EPJ Web of Conferences 71, 00075 (2014)

DOI: 10.1051/epjconf/20147100075

(C) Owned by the authors, published by EDP Sciences, 2014

\title{
New ATLAS results in inclusive searches for supersymmetric squarks and gluinos
}

\author{
Jeanette Miriam Lorenz ${ }^{1, a}$ \\ On behalf of the ATLAS collaboration \\ ${ }^{1}$ Fakultät für Physik \\ LMU München \\ Am Coulombwall 1 \\ D-85748 Garching \\ Germany
}

\begin{abstract}
Despite the absence of experimental evidence, weak-scale supersymmetry remains one of the best motivated and most studied Standard Model extensions. These proceedings summarise recent results from the ATLAS experiment at the LHC on inclusive searches for supersymmetric squarks and gluinos in events containing jets, missing transverse momentum, and possibly isolated leptons in $R$-parity conserving scenarios.
\end{abstract}

\section{Supersymmetric particles produced in strong production}

If Supersymmetry (SUSY) is realized at LHC energies, gluinos and squarks are expected to be produced copiously. Gluinos and squarks will decay either directly or through intermediate particles into jets and the lightest supersymmetric particle (LSP, here assumed to be the lightest neutralino $\tilde{\chi}_{1}^{0}$ ), which is assumed to be stable in these proceedings, as only R-parity conserving scenarios are considered. Being only weakly interacting, the LSP will escape the detector and thus generate a significant amount of missing transverse energy. In more complex decay chains of gluinos or squarks one or more lepton(s) can appear in the final state due to chargino, neutralino or slepton decays. Therefore, the signatures considered here consist of jets, $E_{\mathrm{T}}^{\text {miss }}$ and possibly one or more leptons. Some typical decays are shown in Figure 1. In the following, a selection of analyses targeting this signature are presented, starting with analyses of final states with zero leptons, followed by analyses of final states with one or two leptons. Finally, dedicated analyses looking for stop or sbottom quarks produced in the decay of gluinos will be presented. Model dependent interpretations of the analyses will be given at the end of these proceedings. All of these analyses use the full dataset of $20 \mathrm{fb}^{-1}$ produced in $p p$ collisions at $8 \mathrm{TeV}$ at the LHC [1] and recorded by the ATLAS detector [2].

\section{Final states with jets and $E_{\mathrm{T}}^{\text {miss }}$ but no leptons}

Due to the large branching ratios into final states without leptons, the 0-lepton analysis [3] has large sensitivity to several decay modes of gluinos or squarks. Three different sets of signal regions are

\footnotetext{
ae-mail: Jeanette.Lorenz@physik.uni-muenchen.de
} 

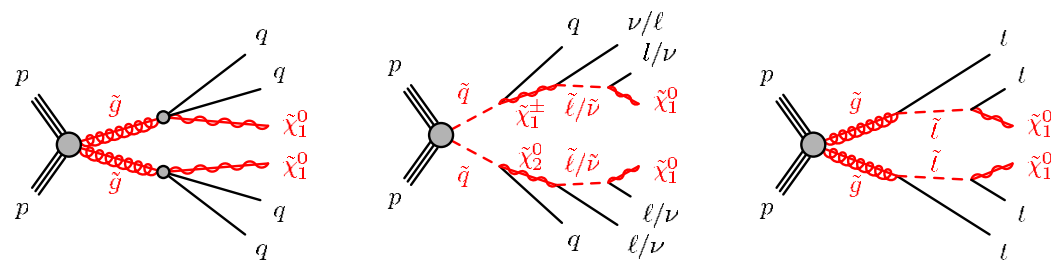

Figure 1. Typical decay chains following a gluino or squark pair production. A direct decay of gluinos into the $\tilde{\chi}_{1}^{0}$ is shown in the left plot, whereas the middle plot shows a squark pair production followed by a two-step decay via intermediate charginos/neutralinos and sleptons/sneutrinos. The right plot shows the decays of gluinos via intermediate stop quarks into the $\tilde{\chi}_{1}^{0}$.
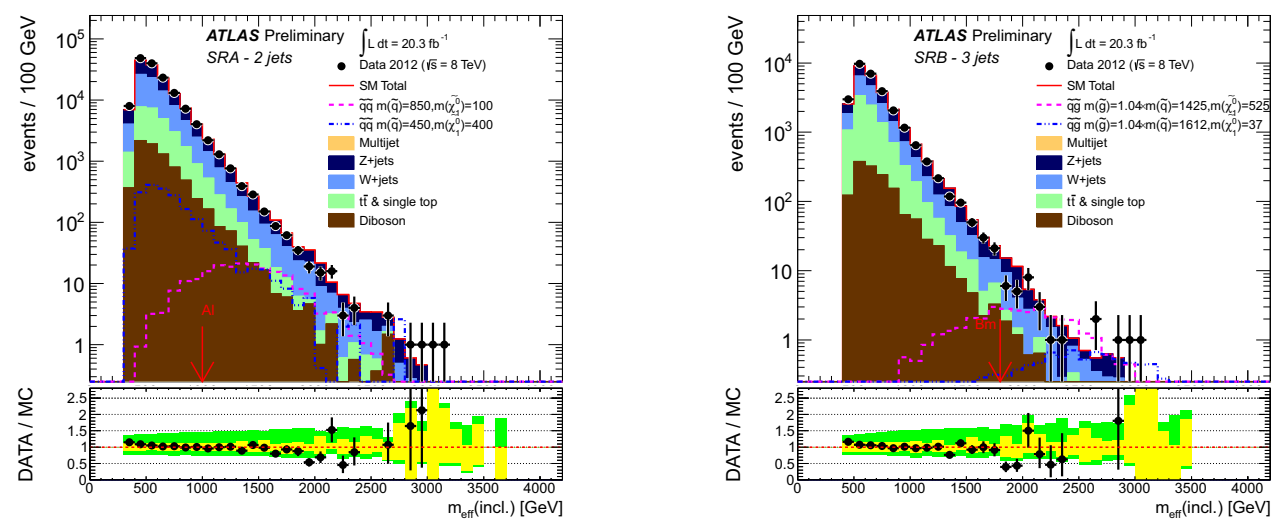

Figure 2. The $m_{\text {eff }}$ distributions are shown before the final cuts on $m_{\text {eff }}$ (which are indicated by an arrow) in signal regions of the 0-lepton analysis requiring two jets (left) or three jets (right) [3]. The $m_{\text {eff }}$ distributions of two signal models are overlaid.

defined with increasing jet multiplicity. In a set of signal regions sensitive to squark pair production two jets are required. In signal regions including also gluino production three jets (looking for squarkgluino pair production) or four, five or six jets are required (gluino pair production or longer decay chains). Further main discriminating variables are $E_{\mathrm{T}}^{\text {miss }}$ (a cut on this variable is already required by the trigger) and the effective mass $\left(m_{\text {eff }}\right)$, constructed as the sum over transverse momenta of all jets and $E_{\mathrm{T}}^{\mathrm{miss}}$. Four different categories of backgrounds are relevant in the signal regions: QCD multi-jet, $Z / \gamma+$ jets, $t \bar{t} /$ single $t$ and $W+$ jets. For each of them a dedicated control region is defined. The $t \bar{t} /$ single $t$ or $W+$ jets backgrounds, for example, are isolated in control regions by requiring a lepton and at least one or no $b$-tagged jet. In each of these control regions, the Monte Carlo (MC) prediction (or the template in the case of the QCD multi-jet background) is normalized to data. This is technically performed by a simultaneous fit to all control regions by the use of a profile log-likelihood method. The background estimate in the signal regions is then obtained by using transfer factors from the control to the signal regions. These transfer factors are taken from $\mathrm{MC}$ or from data in the case of the QCD multi-jet background. No excess beyond the Standard Model expectations has been seen in any 

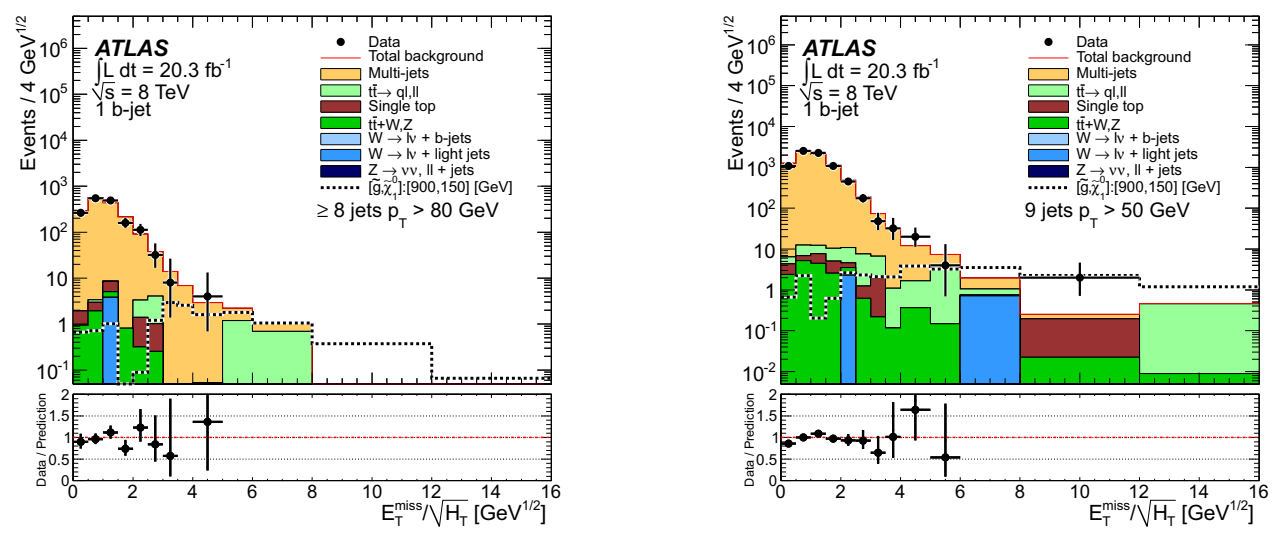

Figure 3. The $E_{\mathrm{T}}^{\text {miss }} / \sqrt{H_{\mathrm{T}}}$ distributions are shown in two signal regions of the multi-jet analysis with requirements for eight jets $\left(p_{\mathrm{T}}>80 \mathrm{GeV}\right)$ including a $b$-tagged jet (left) or for nine jets $\left(p_{\mathrm{T}}>50 \mathrm{GeV}\right)$ including a $b$-tagged jet (right) [4]. As reference, a sumpersymmetric model with pair-produced gluinos is overlaid.

of the signal regions. The $m_{\mathrm{eff}}$ distribution as final discriminating variable is shown before the cut on $m_{\mathrm{eff}}$ in two of the signal regions in Figure 2.

\section{Final states with no leptons and a large number of jets}

The multi-jet analysis [4] focuses on longer supersymmetric decay chains resulting in many jets. Due to the use of a multi-jet trigger, this analysis is able to apply looser cuts on $E_{\mathrm{T}}^{\mathrm{miss}}$ than the 0-lepton analysis described in section 2. Between seven and ten jets are required in the 19 signal regions, which are gathered in three groups. In two groups of signal regions, a further division into categories according to the $b$-jet multiplicity is made, as this increases the sensitivity for supersymmetric models predicting less or more $b$-jets than the Standard Model background. The third group makes use of 'mega-jets', which are constructed by re-clustering the standard anti- $k_{T}$ jets with cone radius of $R=0.4$ into anti- $k_{T}$ jets with cone radius of $R=1.0$. The signal can be discriminated from the background by requiring a larger value for the summed mass of all mega-jets. A cut on the final discriminating variable $E_{\mathrm{T}}^{\text {miss }} / \sqrt{H_{\mathrm{T}}}=4 \sqrt{\mathrm{GeV}}$ is imposed in all signal regions $\left(H_{\mathrm{T}}\right.$ is the sum of all transverse momenta of the jets). The $E_{\mathrm{T}}^{\text {miss }}$ resolution of the detector has been measured to be approximately proportional to $\sqrt{H_{\mathrm{T}}}$ and nearly independent from the jet multiplicity in events dominated by jet activities. Thus, the distribution of $E_{\mathrm{T}}^{\mathrm{miss}} / \sqrt{H_{\mathrm{T}}}$ is nearly invariant under changes of the jet multiplicity. Backgrounds in the signal regions as multi-jet production, $t \bar{t}, W+$ jets or $Z+$ jets production with hadronic decays can be estimated by taking the shape and the normalization of the $E_{\mathrm{T}}^{\text {miss }} / \sqrt{H_{\mathrm{T}}}$ distribution from control regions with lower jet multiplicities or lower $E_{\mathrm{T}}^{\text {miss }} / \sqrt{H_{\mathrm{T}}}$ values. Backgrounds with leptons in the final state can enter the signal regions, if the lepton failed the selection criteria. These are estimated similarly as in the 0-lepton analysis by isolating the backgrounds in control regions and using transfer factors to the signal regions. In all of the signal regions the Standard Model prediction is consistent with the observed data. Figure 3 shows the $E_{\mathrm{T}}^{\text {miss }} / \sqrt{H_{\mathrm{T}}}$ distributions before the final cut in channels requiring eight jets (with $p_{\mathrm{T}}>80 \mathrm{GeV}$ ) or nine jets (with $p_{\mathrm{T}}>50 \mathrm{GeV}$ ) and including a $b$-tagged jet in both cases. 

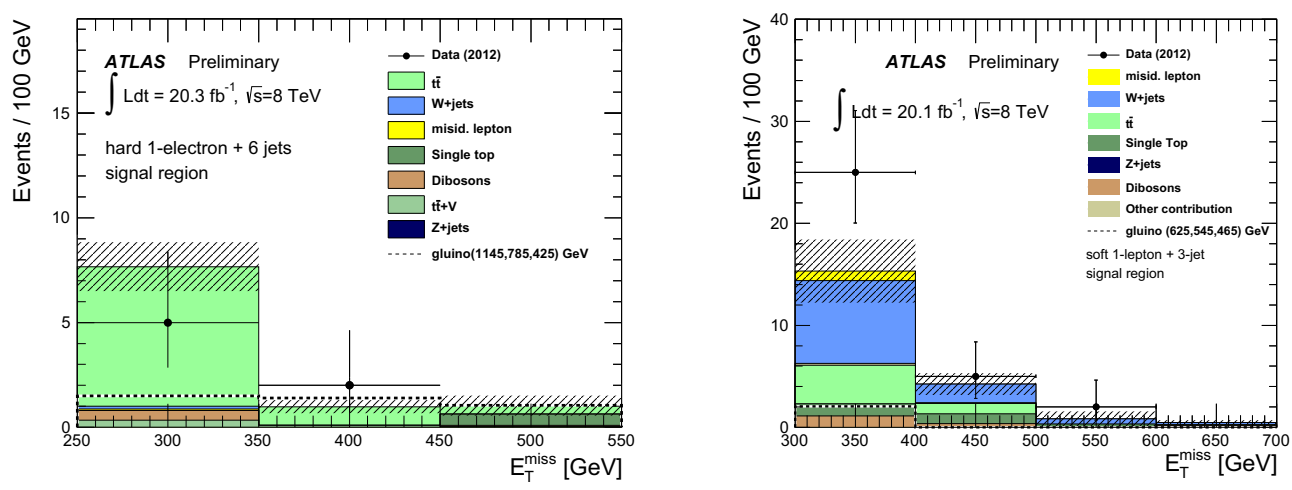

Figure 4. The $E_{\mathrm{T}}^{\text {miss }}$ distribution in a signal region requiring six jets and a hard electron $\left(p_{\mathrm{T}}>25 \mathrm{GeV}\right.$, left) and in a signal region requiring three jets and a soft lepton (electron or muon, $6(\mu) / 10(e)<p_{\mathrm{T}}<25 \mathrm{GeV}$, right) by the 1-lepton analysis [5].

\section{Final states with one lepton}

In contrast to the previous two analyses, the QCD multi-jet background is reduced in the 1-lepton analysis [5] due to the one isolated lepton (electron or muon) requirement. Although the presence of the lepton results in smaller branching ratios, an increase in sensitivity is obtained by the use of the transverse mass of the $E_{\mathrm{T}}^{\text {miss }}$-lepton system, $m_{\mathrm{T}}=\sqrt{2 \cdot E_{\mathrm{T}}^{\text {miss }} \cdot p_{\mathrm{T}} \cdot\left(1-\cos \Delta \phi\left(\vec{E}_{\mathrm{T}}^{\text {miss }}, \vec{p}_{\mathrm{T}}\right)\right)}$, so that the limits of the 1-lepton analysis are comparable with the limits of the 0-lepton and the multijet analyses (as shown in section 7). The 1-lepton analysis splits into two categories, one requiring at least one soft lepton $\left(6(\mu) / 10(e)<p_{\mathrm{T}}<25 \mathrm{GeV}\right)$, the other one requiring a hard lepton with $p_{\mathrm{T}}>25 \mathrm{GeV}$. Whereas the soft lepton signal regions have been designed to provide sensitivity to compressed scenarios with very small mass differences between the gluino/squark and the $\tilde{\chi}_{1}^{0}$, the signal regions requiring a hard lepton show sensitivity for a wide range of models and medium to large mass differences.

The signal regions with a hard lepton requirement use $m_{\mathrm{T}}, E_{\mathrm{T}}^{\mathrm{miss}}$ and $m_{\mathrm{eff}}$ (constructed as sum of the lepton transverse momentum, the transverse momenta of the jets and $E_{\mathrm{T}}^{\mathrm{miss}}$ ) as further discriminating variables between background and signal. Three different sets of signal regions are defined: the signal regions requiring three jets target squark pair production and the signal regions requiring five or six jets provide sensitivity to gluino pair production and longer decay chains. Each signal region exists in an inclusive form, aiming at deriving model independent limits, and in a binned form for interpretation in specific supersymmetric models. In the latter class of signal regions, the sensitivity is increased by explicitly considering the signal and background shape through the binning in $E_{\mathrm{T}}^{\mathrm{miss}}$ or $m_{\mathrm{eff}}$.

The soft lepton signal regions relies on $E_{\mathrm{T}}^{\mathrm{miss}}, m_{\mathrm{T}}$ and $E_{\mathrm{T}}^{\mathrm{miss}} / m_{\mathrm{eff}}$ as main discriminating variables. Two signal regions are defined: one is requiring three jets, the other one five jets.

In both the hard lepton and soft lepton signal regions the dominant backgrounds are $t \bar{t}$ and $W+$ jets production, which are estimated by a semi-data-driven method similarly to the one used in the 0lepton analysis by scaling MC to data in control regions with $b$-jet requirements or $b$-jet vetoes and using transfer factors from MC. The QCD multi-jet background is estimated by a data-driven matrix method, while the smaller backgrounds are taken directly from MC. Background predictions agree 

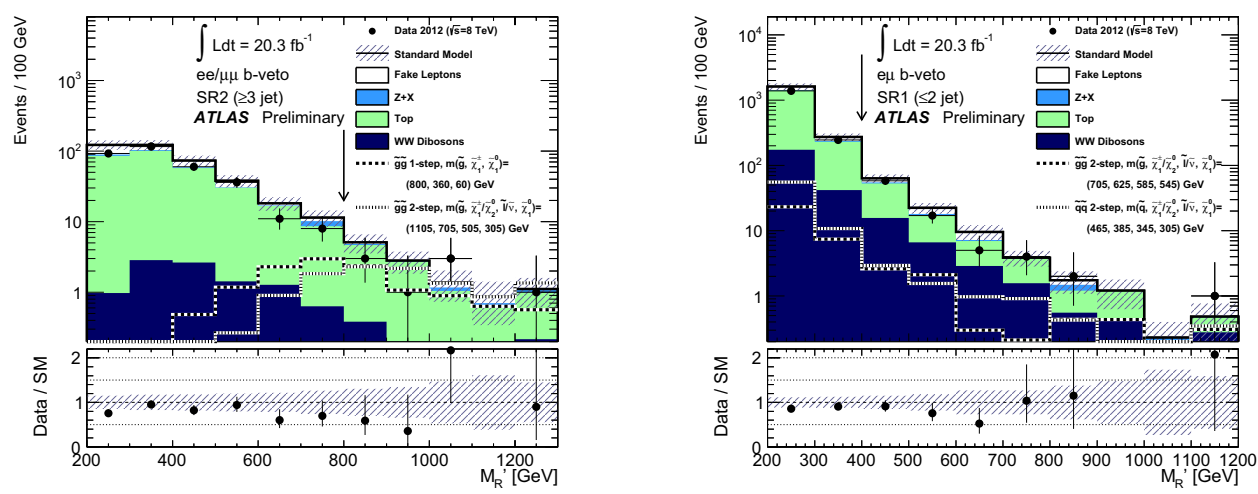

Figure 5. The $M_{R}^{\prime}$ distribution [6] in the signal region by the 2-lepton analysis requiring at least 3 jets (less than 3 jets) is shown before the $M_{R}^{\prime}$ cut (indicated by an arrow) in in the left (right) plot. Two signal models are overlaid.

well with data in the signal regions. Figure 4 shows the $E_{\mathrm{T}}^{\mathrm{miss}}$ and $m_{\mathrm{eff}}$ distributions in selected signal regions.

\section{Final states with two leptons, jets and $E_{\mathrm{T}}^{\text {miss }}$}

The 2-lepton analysis [6] (either electrons or muons) also exploits the longitudinal information in the event. Assuming that the two initially created sparticles are produced at approximately the same mass scale, the decay chains from each sparticle are symmetric in the frame where each of the initial heavy sparticles is at rest. Therefore, all visible particles from one decay chain can be grouped into one mega-jet. Both of the resulting mega-jets will have similar energy. Using the two mega-jets, two event variables can be constructed:

- the characteristic mass of the event:

$$
M_{R}^{\prime}=\sqrt{\left(j_{1, E}+j_{2, E}\right)^{2}-\left(j_{1, L}+j_{2, L}\right)^{2}}
$$

where $E$ is the energy, $L$ the longitudinal component, $j_{1}$ and $j_{2}$ the four-vectors of the two mega-jets

- the transverse information of the event:

$$
M_{\mathrm{T}}^{R}=\sqrt{\frac{\left|\vec{E}_{\mathrm{T}}^{\mathrm{miss}}\right|\left(\left|\vec{j}_{1, \mathrm{~T}}\right|+\left|\vec{j}_{2, \mathrm{~T}}\right|\right)+\vec{E}_{\mathrm{T}}^{\mathrm{miss}} \cdot\left(\vec{j}_{1, \mathrm{~T}}+\vec{j}_{2, \mathrm{~T}}\right)}{2}}
$$

By defining a Razor variable $R=\frac{M_{\mathrm{T}}^{R}}{M_{R}^{\prime}}$ [6], supersymmetric signals can be separated from the Standard Model background, as the background tends to have lower $R$ values, whereas the signal is uniformly distributed in $R$. Using these variables, the 2-lepton analysis defines two signal regions, one requiring less than three jets, the other one requiring at least three jets. In both cases, cuts on $R$ and on $M_{R}^{\prime}$ are applied. In the same-lepton-flavor channels, any event containing a $Z$-boson decaying to leptons is vetoed. Due to this analysis being optimized for models without any explicit $b$-jet appearing in the final states, a veto on $b$-tagged jets is imposed. The backgrounds fall into two categories, 


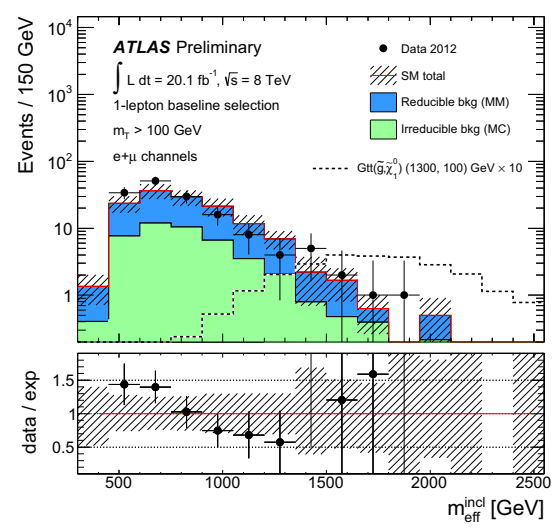

Figure 6. In the $3 b$-jets analysis requiring one lepton, the $m_{\text {eff }}$ distribution is shown after cuts as described in the text [7]. A signal model in a scenario with gluino mediated stop quark production is overlaid.

either belonging to backgrounds with two real leptons, as for example dileptonic $t \bar{t}$ events, or backgrounds with one or more misidentified lepton(s). While the latter is estimated through a data-driven method similar to the 1-lepton analysis, the former one is estimated in control regions defined by lower requirements on $R$ than the signal regions and by demanding the presence of a $b$-tagged jet. The extrapolation to the signal regions is done through transfer factors taken from MC. This analysis does not see any excess beyond Standard Model expectations in the signal regions (as shown in Figure 5).

\section{Analyses with an intermediate sbottom or stop in the decay chain}

In contrast to the previous analyses the 3 b-jets analysis [7] was specifically optimized for a stop or a sbottom quark appearing as intermediate sparticle in the decay chain of an initial gluino. In these cases, multiple $b$-jets are expected in the final states. Thus, the analysis uses signal regions with a requirement for four, six or seven jets of which at least three $b$-tagged jets are required. Further criteria include (depending on the channel): no lepton or one lepton, large $E_{\mathrm{T}}^{\text {miss }}, m_{\mathrm{eff}}, E_{\mathrm{T}}^{\mathrm{miss}} / \sqrt{H_{\mathrm{T}}}$ and $\Delta \phi_{\min }^{4 \mathrm{j}}>0.5$ (where $\Delta \phi_{\min }^{4 \mathrm{j}}$ is defined as the minimum azimuthal separation between any of the four leading jets and $E_{\mathrm{T}}^{\mathrm{miss}}$ ). There are two types of backgrounds: the backgrounds which have three real $b$-jets and the ones in which one or more $b$-jets are misidentified. Examples for the first group contain $t \bar{t}$ events with an additional $b$-jet, examples for the second group are $t \bar{t}$ events without further $b$-jets. Backgrounds with three real $b$-jets are irreducible and therefore taken from MC. Backgrounds without three real $b$-jets are estimated through a matrix method based on the $b$-jet multiplicity and the b-tagging efficiencies. Figure 6 shows the $m_{\text {eff }}$ distribution after cuts on the $(b$-)jet multiplicity, on $E_{\mathrm{T}}^{\text {miss }}>150 \mathrm{GeV}$ and on $m_{\mathrm{T}}>100 \mathrm{GeV}$. This analysis did not observe a disagreement between the background expectations and the observed data in any of the signal regions.

\section{Interpretation in specific models}

As none of the analyses sees an excess, limits are set in a variety of supersymmetric models. Two groups are considered here: simplified models and a MSUGRA model. In simplified models only a 

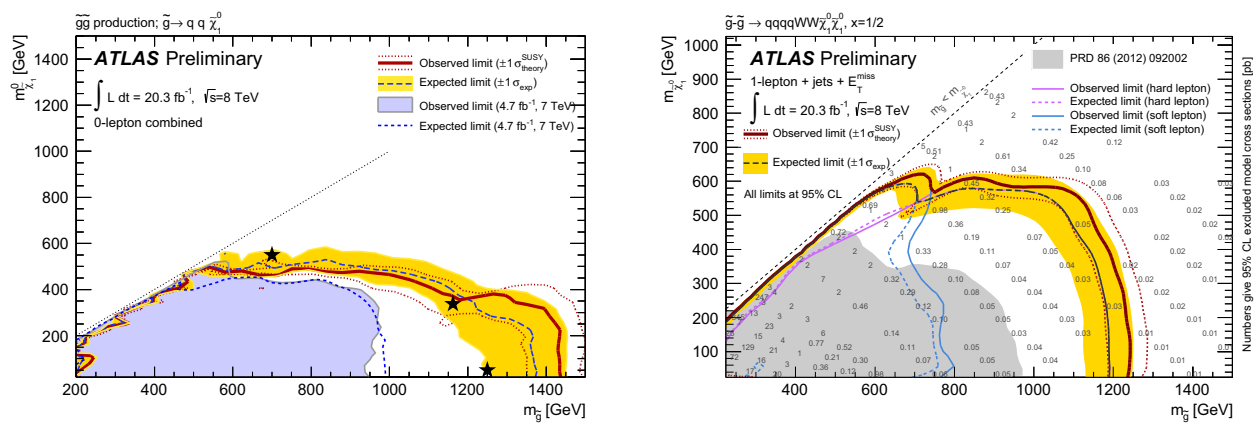

Figure 7. Limits obtained by the 0-lepton analysis in a simplified model with gluino pair production and direct decay to the $\tilde{\chi}_{1}^{0}$ (left) [3]. Limits by the 1-lepton analysis are shown in a simplified model with gluino pair production and decay of each gluino via an intermediate $\tilde{\chi}_{1}^{ \pm}$into the $\tilde{\chi}_{1}^{0}$ (right) [5]. The combination of the limits by the hard and soft lepton analyses is performed by choosing the best expected limit at each point.

particular decay chain and the supersymmetric particles explicitly appearing therein are considered, while all other supersymmetric particles are assumed to be very heavy $(O(5 \mathrm{TeV}))$ and thus decouple. For the considered decay chain a $100 \%$ branching ratio is assumed. Examples include the direct decay of a gluino into a $\tilde{\chi}_{1}^{0}$ by emitting multiple jets. A limit by the 0 -lepton analysis is shown in Figure 7, where gluino masses up to $1350 \mathrm{GeV}$ can be excluded for very small $\tilde{\chi}_{1}^{0}$ masses. Another example is the decay of pair-produced gluinos or squarks via an intermediate chargino into the $\tilde{\chi}_{1}^{0}$. Limits are shown in Figures 7 and 8 for the 1-lepton analysis. In these models, gluino masses up to $1.18 \mathrm{TeV}$ and squark masses up to $700 \mathrm{GeV}$ are excluded for very small $\tilde{\chi}_{1}^{0}$ masses. Close to the mass diagonal, gluino masses up to $700 \mathrm{GeV}$ can be excluded for a mass difference of $25 \mathrm{GeV}$ between the $\tilde{\chi}_{1}^{0}$ and the $\tilde{g}$. Similar limits (Figure 8) can be obtained in more complex decay chains with two steps with intermediate charginos/neutralinos. In the case of an intermediate stop quark, which is assumed to be off-shell, gluino masses up to $1350 \mathrm{GeV}$ can be excluded, as shown in Figure 9. In a MSUGRA grid with parameters $\tan \beta=30, A_{0}=-2 m_{0}$ and $\mu>0$, gluino masses up to $1.3 \mathrm{TeV}$ can be excluded for any $m_{0}$ value.

\section{References}

[1] Lyndon Evans and Philip Bryant, LHC Machine, JINST 3 S08001 (2008).

[2] ATLAS Collaboration, The ATLAS Experiment at the CERN Large Hadron Collider, JINST 3 S08003 (2008).

[3] ATLAS Collaboration, Search for squarks and gluinos with the ATLAS detector in final states with jets and missing transverse momentum and $20.3 \mathrm{fb}^{-1}$ of $\sqrt{\mathrm{s}}=8 \mathrm{TeV}$ proton-proton collision data, ATLAS-CONF-2013-047, http://cds . cern. ch/record/1547563.

[4] ATLAS Collaboration, Search for new phenomena in final states with large jet multiplicities and missing transverse momentum at $\sqrt{s}=8 \mathrm{TeV}$ proton-proton collisions using the ATLAS experiment, arXiv:1308.1841, accepted by JHEP.

[5] ATLAS Collaboration, Search for squarks and gluinos in events with isolated leptons, jets and missing transverse momentum at $\sqrt{s}=8 \mathrm{TeV}$ with the ATLAS detector, ATLAS-CONF-2013-062, http: //cdsweb. cern.ch/record/1557779. 
EPJ Web of Conferences
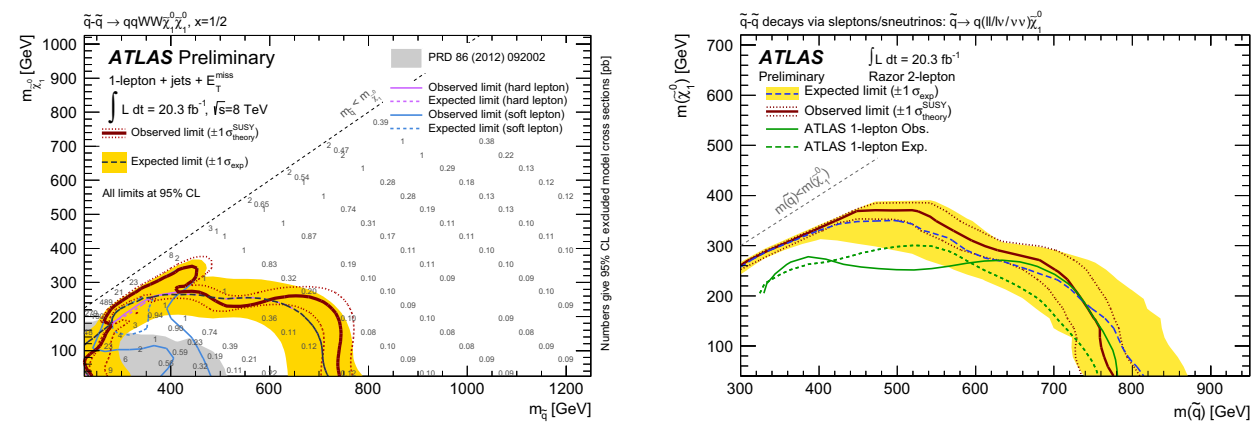

Figure 8. Limits in simplified models with squark pair production and a decay through an intermediate $\tilde{\chi}_{1}^{ \pm}$into the $\tilde{\chi}_{1}^{0}$ as obtained by the 1-lepton analysis (left) [5] or a decay through intermediate charginos/neutralinos and sleptons/sneutrinos as derived by the 2-lepton analysis (right) [6].
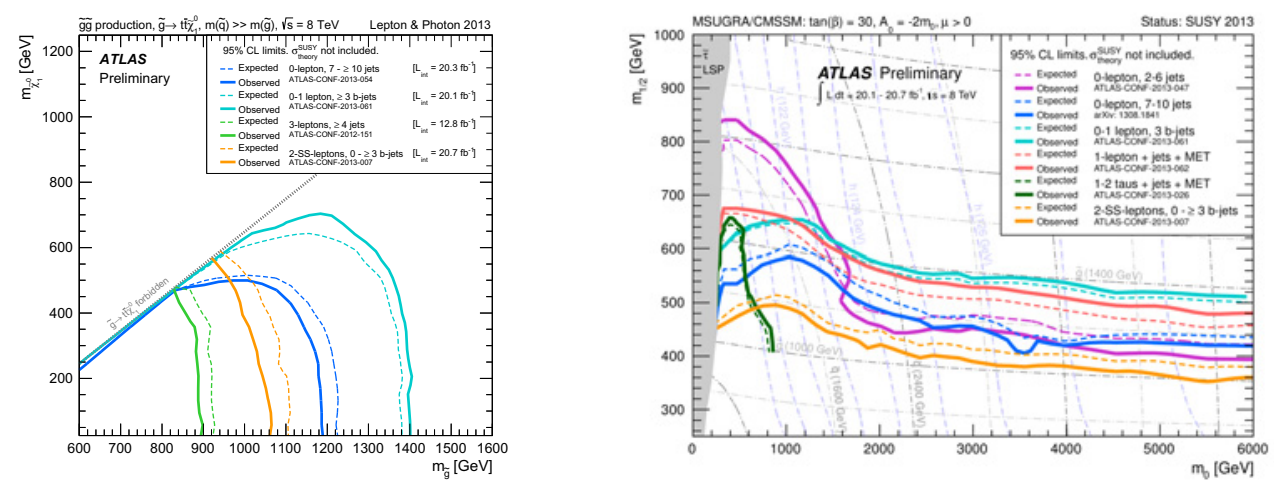

Figure 9. Limits by various analyses in a simplified model with gluino pair production and decay of each gluino via an off-shell stop into the $\tilde{\chi}_{1}^{0}$ (left) [8]. Limits by multiple analyses in the MSUGRA model with parameters as defined in the text (right) [9].

[6] ATLAS Collaboration, Search for strongly produced supersymmetric particles in decays with two leptons at $\sqrt{s}=8 \mathrm{TeV}$, ATLAS-CONF-2013-089, http://cds.cern.ch/record/1595272

[7] ATLAS Collaboration, Search for strong production of supersymmetric particles in final states with missing transverse momentum and at least three b-jets using $20.1 \mathrm{fb}^{-1}$ of pp collisions at $\sqrt{s}=8 \mathrm{TeV}$ with the ATLAS Detector, ATLAS-CONF-2013-061, http://cds. cern.ch/record/1557778.

[8] http://twiki.cern.ch/twiki/pub/AtlasPublic/CombinedSummaryPlots/ ATLAS_SUSY_Gtt_lp13.pdf.

[9] http://twiki.cern.ch/twiki/pub/AtlasPublic/CombinedSummaryPlots/ ATLAS_SUSY_MSUGRA_CMSSM_susy13-1.pdf. 